\title{
Imagen corporal, IMC, afrontamiento, depresión y riesgo de TCA en jóvenes universitarios
}

\author{
Lina-María Hernández-Cortés ${ }^{1}$ y Constanza Londoño Pérez ${ }^{2 *}$
}

${ }^{1}$ Universidad de Ibagué (Colombia)

${ }^{2}$ Universidad Católica de Colombia

\begin{abstract}
Resumen: El presente estudio descriptivo correlacional pretendió determinar la relación entre estilo de afrontamiento, historia psiquiátrica familiar, salud percibida, IMC, género, percepción de la figura y depresión y el riesgo de padecer un trastorno de conducta alimentaria. Participaron 417 estudiantes universitarios que se seleccionaron de manera no aleatorizada estratificada con grupos de conveniencia. Para obtener los datos se utilizó la Ficha de Registro de Información General, la Escala Abreviada de las actitudes alimentarias EAT -26-M, el Cuestionario de Formas de Afrontamiento, el Inventario de Depresión de Beck, la Prueba de Optimismo Disposicional y el Registro de Percepción de la Figura. El análisis de datos se llevo a cabo mediante el uso de ecuaciones estructurales. Se concluyó que la interacción de las variables antecedentes psicológicos familiares, género, deseo de disminución del peso, satisfacción con la imagen corporal, estilo de afrontamiento evitativo y depresión en conjunto predicen el riesgo de padecer un TCA, se discuten los hallazgos y se evidencian las limitaciones del presente estudio.

Palabras clave: Afrontamiento; depresión; figura corporal percibida; trastornos de la conducta alimentaria; jóvenes.
\end{abstract}

\section{Introducción}

Históricamente se ha considerado que la alimentación está ligada al prestigio social y al estatus; igualmente, los modos de alimentación constituyen un medio de afirmación y aumento de prestigio social; en la actualidad existe gran preocupación por la comida, las dietas, la imagen y la figura (Waller \& Sheffield, 2008; Lugli \& Vivas, 2006), lo cual ha llevado a que las preferencias sociales por la delgadez y el estilo de vida dirigido a ésta afecten notoriamente la conducta alimentaria y las actividades de las personas en torno a la comida. Los Trastornos de la Conducta Alimentaria (TCA) se caracterizan por la alteración del comportamiento de ingesta de alimentos del individuo que está acompañado de una serie de conflictos psicosociales y su resolución está inevitablemente condicionada por el logro y/o persistencia de un estado de delgadez (Maor, Sayag, Dahan \& Hermoni, 2006; Santacoloma \& Carmona, 2009, López, Molano y Piñeros 2010). Estos trastornos no son una patología moderna, ni un problema nuevo ya que algunos fueron conocidos y descritos en la antigüedad; lo novedoso, en cambio, es la frecuencia con la que se están presentando en las últimas décadas en las sociedades industrializadas y el cambio de actitudes que ésta ha tenido en los conceptos de salud y de ideal estético (Lugli \& Vivas, 2006; Acosta, Llopis, Gómez \& Pineda, 2005; Martínez-González \& De Irala, 2003).

* Dirección para correspondencia [Correspondence address]:

Constanza Londoño Pérez. Grupo de Investigación ENLACE, Facultad de Psicología. Universidad Católica de Colombia. Avenida Caracas No. 46 - 44 piso 1 Postgrados Facultad de Psicología.

E-mail: clondono@ucatolica.edu.co
Title: Body Image, CMI, coping, depression and BED risk in young univesitaries.

Abstract: The main objective of this descriptive correlation study was to determine the relation between coping styles, psychiatric family history, perceived health levels; body mass index, gender, body figure perception, depression and the risk of develop an eating behavior disorders. The sample was composed with 300 university students and those students were selected by two methods: stratified and convenience groups. In order to obtain data, it has been administered the General Information Checklist, Eating Behaviors Brief Scale EAT - 26 M, Coping Styles Questionnaire, Beck Depression Inventory, Dispositional Optimism Test and the Body Perception Checklist. For data analysis, it has been used structural equations. Results showed that psychiatric family history, gender, desire to reduce weight, satisfaction with body image, avoidance coping style and depression predicts the risk of develop an eating behavior disorder. Results were discussed and some limitations of this research were pointed out.

Keys words: Coping; depression; perceived corporal image; eating behavior disorders; young.

Los TCA han alcanzado proporciones preocupantes entre la población de los llamados países desarrollados, principalmente entre mujeres adolescentes y adultas jóvenes de nivel socio económico medio alto. Precisar los datos epidemiológicos reales no ha sido fácil puesto, que en una gran mayoría de casos, estos trastornos no son diagnosticados debido a que las pacientes rara vez revelan o comunican sus síntomas, ya sea al médico o a su familia. Fandiño, Giraldo, Martínez, Aux \& Espinosa (2007) sugieren que aproximadamente la incidencia de la Anorexia (AN) en mujeres menores de 25 años es de 20 a 30 por 100.000 habitantes, con una prevalencia del $0.5 \%$ al $1 \%$ y la incidencia de la Bulimina (BN) entre el 1 y el 13\%, con una prevalencia entre el 1 y el $20 \%$; cifras concordantes con las publicadas por la Organización Mundial de la Salud (OMS) en 2005.

En el último Estudio Nacional de Salud Mental para Colombia 2003, adelantado por la fundación FES para el Ministerio de Protección Social se presentó un informe preliminar de los datos, que arrojó una prevalencia de vida de "Trastornos de la alimentación" de $0.0 \%$ (varones) y $0.5 \%$ (mujeres). En dicho estudio estos trastornos se encuentran clasificados dentro de los trastornos de impulsividad. Previamente, en el Segundo Estudio Nacional de Salud Mental de 1997 encontró una prevalencia de Anorexia de 2.4\% y de Bulimia de $0.2 \%$ para mujeres (Ministerio de Protección Social, Ministerio de Educación Nacional [MEN] 2003).

En el trabajo Prevalencia de trastornos del comportamiento alimentario en estudiantes de la Universidad Nacional de Colombia entre julio de 1994 y diciembre de 1995 por Ángel, Vásquez, Martínez, Chavarro \& García 2000; y en el de Castrillón, Luna \& Aguirre-Acevedo, (2007) se concluyó que la prevalencia de los trastornos de la alimentación en el 
grupo universitario estudiado y residente en la ciudad de Bogotá es similar a la informada en países desarrollados. En otro análisis de la prevalencia de anorexibulimia en mujeres adolescentes realizado recientemente en Manizales, Cano et al. (2007) encontraron una probabilidad de tener un TCA del $35 \%$ y una prevalencia total cercana al $17 \%$, una prevalencia de AN del $0.9 \%$, de BN del 2.6\% y de TCA no especificados del $14.9 \%$. Se mostró cómo para Medellín, una tercera parte de las mujeres adolescentes estaba en riesgo de sufrir un TCA, y de éstas, el $15.1 \%$ ya tienen un trastorno sub clínico, sin distinción de condición social, grado escolar ó tipo de colegio; con resultados similares en Cali (Fandiño et al., 2007).

Desde el punto de vista psíquico-médico-social existe una gran preocupación por abordar la problemática de los trastornos del comportamiento alimentario; su incremento en los últimos años ha conducido a que durante la última década se haya producido una gran proliferación de investigaciones a fin de poder determinar, de una parte, los principales factores implicados en la adquisición y mantenimiento de estos trastornos de conducta alimentaria y, de otra, desarrollar instrumentos de evaluación y procedimientos terapéuticos efectivos para poder tratar los TCA y potenciar las vías para su prevención.

Respecto a los factores implicados en la adquisición y mantenimiento de los TCA, diversos estudios evidencian la existencia de ciertos indicadores de riesgo para el desarrollo de los mismos. Moreno \& Ortiz (2009) identificaron como factores de riesgo para que adolescentes entre los 12 y los 15 años padezcan un TCA, el perfeccionismo, el riesgo de enfermedad psíquica (en pacientes y familiares) y la autoevaluación negativa.

León et al., (2005) plantean que hay una gran variedad de factores de riesgo clasificados en tres grupos: predisponentes, precipitantes y perpetuantes. Los factores predisponentes actúan desde el inicio de la vida y condicionan la vulnerabilidad de un individuo a padecer el trastorno; tales como el sobrepeso, la baja autoestima (Engler, Crowther, Dalton, \& Sanftner, 2006, De la Fuente, García \& Salvador 2009 Pérez \& Romero, 2008), inseguridad e introversión, obesidad y perfeccionismo, (identificado como un predictor de síntomas bulímicos y un factor de mantenimiento entre individuos sintomáticos (Azevedo et al., 2010; Engler et al., 2006), los prejuicios sociales respecto a la obesidad, malos hábitos alimenticios en la familia, y la presión familiar por la figura (Ghaderi, 2003), entre otros. Los factores precipitantes suelen acontecer inmediatamente antes del inicio del trastorno y comprenden los comentarios negativos sobre la figura, la insatisfacción general con uno mismo (Grylli et al., 2005), el estrés (Engler et al., 2006), los conflictos familiares y los fracasos amorosos, entre otros; y los factores perpetuantes son los que mantienen o prolongan la evolución del trastorno, una vez se ha desencadenado el proceso, como por ejemplo una alimentación incorrecta que genera desnutrición y refuerza la imagen corporal negativa, fluctuación de los estados del ánimo, ansiedad, depresión (Bardone \& Fitz- simmonsa 2011; Davids, DGreen, Hallengren \& Scott; 2009; Markey \& Vanderwal, 2007; Moreno, Ortega \& Rodríguez, 2009) e irascibilidad, entre otros (Largo, 2003, García, 2003).

Si bien se han sugerido muchas variables como factores de riesgo de los TCA, sólo en pocas ocasiones se han establecido relaciones de tipo causal o se esclarece respecto a qué tanto un aspecto es factor de riesgo para el desarrollo de un TCA o es el resultado o consecuencia de la evolución del mismo. Dancyger, Fornaria, Sciontic, Sundaya \& Wisotskya (2009), Ghaderi (2003) y López et al. (2010), en sus investigaciones, identificaron un Perfil de Riesgo para el desarrollo de los Trastornos de Conducta Alimentaria: baja autoestima, bajo soporte social familiar percibido (Kluck (2010), altos niveles de preocupación por la figura y uso de una estrategia evitativa para afrontar las situaciones de estrés.

Engler et al. (2006), González, Oudhof, Rodríguez \& Unikel (2010) y Bolaños \& Jáuregui (2010) y proponen que las mujeres jóvenes que experimentan baja autoestima pueden ser más susceptibles a la presión sociocultural que promueve un ideal de belleza, poco realista, trasmitido por el medio, la familia y los pares. Estudios como este han aportado a la explicación del desarrollo y mantenimiento del trastorno ya que han identificado el papel del grupo familiar y las pautas culturales. Además dejan interrogantes respecto a, ¿qué aspectos protectores poseen las jóvenes respecto de los TCA, aún cuando están expuestas a patrones culturales similares? o ¿qué habilidades cognitivas trasmiten las familias a sus hijos que los protegen frente a la posibilidad de desarrollar un TCA o les aumenta el riesgo de padecerlo?

Varios estudios han corroborado la relación estrecha que existe entre los recursos de afrontamiento que posee la persona y el riesgo de sufrir un TCA, especialmente si se tiene un estilo negativo o pasivo frente a las situaciones. Entre estos trabajos se encuentran el de Corstorphine, Mountford, Tomlinson, Waller \& Meyer (2007), quienes desarrollaron una medida de tolerancia al estrés para validarla clínicamente con los trastornos de la conducta alimentaria; tomaron como muestra 72 mujeres con diagnóstico para trastorno de conducta alimentaria según criterios del DSM IV y 62 mujeres sin historia del trastorno, y evidenciaron que la aparición de los TCA está asociada con el afrontamiento evitativo. Por su parte Grylli, Wagner, Hafferl-Gattermayer, Schober, \& Karwautz (2005) evaluaron los estilos de afrontamiento y la calidad de vida en jóvenes con diabetes tipo I con y sin TCA y encontraron que las estrategias afrontamiento evitativas estaban relacionadas positivamente con la calidad de vida reportada y con el control metabólico alcanzado por los pacientes. Así mismo Carver \& Connor-Smith (2010) notaron que el afrontamiento comprometido con la solución del problema parecía estar asociado con mejor salud mental en población general.

Estos informes han sido decisivos en la definición de los perfiles de riesgo de TCA, sin embargo tienen una clara limitación, ya que centran su atención en los estilos evitativos a tal punto que usan los cuestionarios de afrontamiento de manera fragmentada para evaluar casi exclusivamente otras 
estrategias; por tanto es preciso describir y comprender la relación de protección o de riesgo que pueden tener otros estilos de afrontamiento que no han sido suficientemente indagados. Por ejemplo, hay que explorar lo que ocurre con otras estrategias de afrontamiento positivas y determinar si las personas con TCA, no hacen uso de éstas.

En la misma dirección podría pensarse que afrontamiento de corte optimista como el optimismo disposicional propuesto por Seligman (2003), Taylor \& Stanton (2007), Jaser et al. (2007), que ha sido considerado como factor protector y potenciador de la salud mental y física, puede ser considerado entre los factores que protegen a las personas de desarrollar un TCA, ya que resulta ser una estrategia del todo opuesta a la evitativa típicamente asociada a estos trastornos. Entre los estudios que corroboran el papel positivo del optimismo sobre la salud se encuentran el realizado por Tusaie y Patterson, 2006, en el cual se encontró que el optimismo disposicional es un factor que contribuye a la resiliencia por ser cognitivamente influyente en los adolescentes para moderar el efecto de los estresores de la vida (Velasco, Botero \& Londoño, 2011).

En los estudios antes mencionados se usó indiferenciadamente el Test de Orientación Vital - LOT para medir optimismo como un rasgo y como un estilo de pensamiento, sin que realmente una escala tan breve permita hacer un análisis discriminativo al respecto o que facilite la descripción del optimismo ya que las preguntas están elaboradas de forma general y con problemas en el uso del lenguaje. A este respecto, Fischer \& Chalmers (2008) desarrollaron una investigación Meta analítica de los niveles de optimismo disposicional con el propósito de responder al interrogante de si existen diferencias culturales de optimismo y cómo ellas pueden ser explicadas. El análisis indicó efectos de variabilidad cultural sobre los niveles de optimismo a la vez que hizo evidente una falencia frente a la medición del optimismo por medio del LOT, pues este instrumento ha sido criticado por usar un lenguaje coloquial e incluyen ítems que no miden directamente optimismo.

Estos estudios nos llevan a entender las facultades del optimismo como estrategia de afrontamiento que provee a los individuos de un repertorio cognitivo y comportamental para responder a las situaciones adversas y nos enfrentan al reto tanto de buscar otras formas de medición del optimismo como de pensar en otros estudios que relacione el optimismo con situaciones clínicas y de salud, como el caso de los trastornos de la conducta alimentaria. Respecto del control emocional, Markey \& Vanderwal, (2007) evaluaron el papel de la inteligencia emocional y otras variables de regulación de emoción en la relación entre afecto negativo y sintomatología bulímica en una muestra de 154 mujeres estudiantes universitarias del medio oeste de Estados Unidos y encontraron que las emociones no reguladas están relacionadas con el desarrollo y mantenimiento de estos trastornos.

Desde hace aproximadamente una década, los Trastornos de la Conducta Alimentaria (TCA) generan gran preocupación a la comunidad médica colombiana, ya sea por la gravedad de sus manifestaciones más extremas, por el incremento en su incidencia y prevalencia, y por los factores de riesgo de tipo cultural presentes en algunas regiones del país. En el país se han desarrollado estudios que aportan a la validación de instrumentos que permitan acercarse a las características y prevalencia del trastornos de TCA en la población colombiana ya que hasta el momento no se contaba con un instrumento psicométricamente válido, que facilitara la evaluación en este grupo específico, ya fuera con fines de diagnóstico para intervención o de detección temprana de casos para desarrollar los programas oportunos de prevención; entre ellos el de Castrillón et al. (2007), y el de Castrillón, Luna \& Aguirre-Acevedo (2007).

Castrillón et al. (2007) han trabajado en la validación del Cuestionario de la figura corporal (Body Shape Questionnaire) BSQ para la población colombiana, con un grupo de 1939 mujeres (niñas, preadolescentes y adolescentes escolarizadas, con edades entre los 9 y 19 años, de 7 colegios (4 públicos y 3 privados) de la ciudad de Popayán. El instrumento evalúa las alteraciones actitudinales de la imagen corporal mediante dos factores: Insatisfacción corporal y Preocupación por el peso y se ha considerado una herramienta útil para identificar individuos en riesgo de desarrollar un trastorno de comportamiento alimentario.

En otro estudio, Castrillón et al. (2007) validaron la Escala abreviada y modificada de las actitudes alimentarias (Escala Abreviada de las Actitudes Alimentarias) EAT-26 para la población colombiana, con un grupo de 2.523 niños y niñas (580 niños y 1.943 niñas), preadolescentes y adolescentes escolarizados en 7 colegios (4 públicos y 3 privados) en edades comprendidas entre los 9 y 19 años, elegidos de forma aleatoria en la ciudad de Popayán. El objetivo principal del test es identificar síntomas y preocupaciones características de los trastornos de la conducta alimentaria en muestras no clínicas. Para Colombia los factores encontrados fueron: 1. preocupación por la ganancia de peso corporal, 2. Preocupación por la cantidad de calorías contenidas en los alimentos, 3. preocupación obsesiva por la comida, 4. bulimia, 5. preocupación social por la ganancia de peso.

Ya que estos estudios fueron desarrollados con fines psicométricos, aún queda la necesidad de identificar los factores de riesgo asociados a la aparición del trastorno que faciliten la comprensión de su adquisición y permitan desarrollar estudios que aporten a la prevención del mismo en el mundo. Adicionalmente, en la revisión de antecedentes empíricos con fines diferentes a los de desarrollo y validación de instrumentos no se encuentra reporte de estudios que hayan indagado específicamente la relación entre las variables psicológicas como afrontamiento y depresión y el riesgo de padecer un trastorno de la conducta alimentaria, de forma simultánea, específicamente en población colombiana. Tanto en el área de promoción en salud y prevención de los TCA, así como también en la detección de personas que se encuentren en situación de riesgo de padecer un TCA y en la observación de la evolución clínica de pacientes en tratamiento para los TCA es importante ampliar la información 
sobre la influencia del conjunto de variables incluidas en esta investigación sobre los trastornos de la conducta alimentaria.

$\mathrm{Si}$ bien estas variables han sido relacionadas de manera independiente con la sintomatología, adquisición y mantenimiento de los TCA, en esta propuesta de investigación se están considerando de manera conjunta para formular un modelo de riesgo más completo, que aporte a la explicación y conocimiento de este problema en una población colombiana que a futuro permita diseñar programas de tratamiento y de prevención más efectivos; ya que propuestas como ésta pueden aportar a la comprensión de un problema de interés académico y social, ya sea en la generación de nuevo conocimiento clave en el abordaje de estos trastornos o en la formulación de nuevas preguntas de investigación.

Por tanto el presente trabajo tenía como objetivo indagar si el estilo de afrontamiento, la historia psiquiátrica familiar, la salud percibida, el Índice de Masa Corporal, el género, la percepción de la figura y la depresión están asociados al riesgo de padecer un trastorno de la conducta alimentaria en estudiantes universitarios, entre los 16 y los 25 años. La cuestión a resolver es si un modelo predictivo conformado por variables como el estilo de afrontamiento, la historia psiquiátrica familiar, la salud percibida, el Índice de Masa Corporal, el género, la percepción de la figura y la depresión; explican al menos el 30\% de la varianza del riesgo de padecer un TCA en estudiantes universitarios. Cabe notar que en la categoría de riesgo se incluyen a los jóvenes con los distintos niveles de riesgo (moderado y alto) teniendo en cuenta la tendencia al incremento de los TCA y la necesidad de actuar oportunamente.

\section{Método}

Esta investigación es de corte descriptivo correlacional con análisis multivariante. La muestra estuvo conformada por 417 estudiantes de dos Universidades ubicadas en las ciudades de Bogotá e Ibagué - Colombia. La muestra se conformó teniendo en cuenta el tamaño del universo poblacional correspondiente a cada institución, y de forma estratificada para garantizar la inclusión de estudiantes de diversas carreras y de todos los semestres. En la institución ubicada en Bogotá el número de sujetos correspondió al menos a $11 \%$ del total de la población matriculada en las carreras incluidas en el estudio, y en la institución ubicada en la ciudad de Ibagué el número de sujetos correspondió al menos al $16 \%$ del total de la población matriculada en las carreras incluidas en el estudio. Los estudiantes fueron abordados en clase previa autorización de la Universidad y la Facultad y la firma del consentimiento informado por cada uno de los participantes. Previo a la firma del consentimiento informado se presentó la ficha técnica de la investigación, aclarando los objetivos de la misma, el manejo ético y confidencial de la información, haciendo énfasis en la voluntariedad de la participación y en la posibilidad de conocer los resultados individuales. En todo momento se facilitó la salida de las aulas a las personas que desistieron de participar.

\section{Instrumentos}

\section{Ficha de Registro de Información General}

Esta incluyó los datos de edad, sexo, peso actual, talla; preguntas de salud percibida respecto a la calidad, a la atribución de diagnóstico y a experiencia de síntomas o dolencias actuales y reporte sobre la historia psiquiátrica familiar.

Registro de percepción de la figura

Este cuestionario incluye la estimación de la imagen corporal detallada, la persona debe elegir la silueta que a su juicio mejor representa su imagen; incluye un juego de nueve siluetas, en rangos que van desde muy delgada hasta extremadamente obesa. De manera complementaria la persona valora el peso actual en kilogramos y la talla actual en metros, y se compara con la medida real obtenida en un proceso de contraste. La magnitud de la diferencia entre las medidas de peso y talla son tomadas en cuenta como indicadores de dificultad para asumir el peso real y/o la alteración de la figura corporal. Validado para población de habla hispana por Lee (2005).

Escala Abreviada de las actitudes alimentarias EAT -26-M (Garner, Olmsted, Bohr, \& Garfinkel, 1982)

La escala está compuesta por 26 ítems (EAT-26), está basada en un factor de análisis de la escala original (EAT40). El EAT-26 tiene una elevada correlación con el EAT-40 $(r=0.98)$. De esta nueva prueba abreviada se obtuvieron a su vez tres subescalas mediante un análisis factorial las cuales están relacionadas de forma significativa con variables como bulimia, peso corporal, imagen corporal y síntomas psicosociales. Los factores se agruparon en 5 áreas específicas; el primer factor evalúa preocupación por la ganancia del peso corporal; el segundo factor se refiere a preocupación por la cantidad de calorías contenidas en los alimentos; el tercer factor evalúa preocupación obsesiva por la comida; el cuarto factor mide bulimia, y el quinto factor mide preocupación social por la ganancia de peso. Esta escala ha sido validada para población colombiana por Castrillón et al. (2007), mostrando poseer una adecuada consistencia, con una confiabilidad por alpha de Cronbach con un coeficiente de correlación intra clase con un resultado de 0.87 .

\section{El Cuestionario de formas de afrontamiento}

Este cuestionario fue desarrollado por Folkman \& Lazarus (1986), consta de 66 ítems agrupados en ocho subescalas o factores, los cuales miden cogniciones y comportamientos utilizados por individuos para afrontar el estrés de la vida diaria. Los estilos de afrontamiento que mide son confrontación, distanciamiento, autocontrol, búsqueda de apoyo social, aceptación de responsabilidad, evitación, planificación de soluciones y reevaluación positiva. El cuestionario con- 
tiene una escala tipo Likert con cuatro opciones de respuesta y ha sido adaptado a población española por Vitaliano, Russo, Carr, Maiuro \& Becker (1985) y por Londoño \& Archila (2004) para población colombiana que se tomará para el estudio, cuya confiabilidad es mayor a 0.7 según el alpha de Cronbach.

\section{Inventario de Depresión de Beck}

Este cuestionario desarrollado por Beck en 1979, consta de 21 ítems para evaluar la intensidad de la depresión; en cada uno de ellos el sujeto tiene que elegir una frase entre un conjunto de cuatro alternativas, siempre ordenadas por su gravedad, la que más se aproxime a su estado medio durante la última semana, incluyendo el día en el que contesta el inventario. Los ítems están graduados en una escala de 0-3 correspondientes a cada una de las afirmaciones, de manera que las puntuaciones totales pueden variar entre 0 y 63 , con las puntuaciones más altas reflejando los niveles más altos de depresión. El BDI ha sido adaptado y validado al castellano por Vázquez \& Sanz (1991), Este es el instrumento de autoinforme más utilizado internacionalmentete para cuantificar los síntomas depresivos en poblaciones normales y clínicas, tanto en la práctica profesional como en la investigativa, mostrando una alta correlación con el diagnóstico a través de la entrevista. Su fiabilidad informada en población española es de 0.83 .

\section{La Prueba de optimismo disposicional}

Desarrollada por Londoño, Hernández, Alejo, \& Pulido (2013), cuyo objetivo es el de evaluar la tendencia personal hacia el optimismo disposicional a través de ítems en los que se presentan diversas situaciones propias de la vida adolescente; entre las opciones de respuesta se encuentran tres alternativas: una optimista, una de sesgo optimista y una pesimista. La prueba consta de 20 ítems seleccionados de un total de 50. Los ítems seleccionados mostraron un buen comportamiento psicométrico y todos aportaron de manera importante al constructo; mostrando una confiabilidad alta de 0.85. Para su calificación se mira el número de respuestas a cada una de las alternativas, que representan las tres tendencias a responder ante situaciones de la vida, para determinar cuál es el estilo predominante en cada persona o si existe un posible perfil mixto, que no responde a un estilo universal de respuesta, el cual sería permanente a todas las áreas de la vi$\mathrm{da}$, si no que por el contrario alude a un estilo situacional que sería cambiante de acuerdo a las características de la situación y al área de la vida en la que esta se presenta.

\section{Procedimiento}

La investigación se realizó en tres fases: primero se solicitó la autorización a las directivas de la institución para la aplicación de los instrumentos, posteriormente se realizó el contacto con los directores de programa y con los docentes de las clases en las cuales se aplicaron los instrumentos, para acordar el momento de la aplicación. En la segunda fase se recolectó la información. Antes de iniciar la aplicación de instrumentos los participantes leyeron, firmaron y devolvieron el formato de consentimiento informado, y a quienes expresaron no querer participar se les solicitó que esperaran fuera del aula. Posterior al aplicación de los instrumentos, y en un salón contiguo se midió en cada participante su peso y talla. En la tercera fase se procedió al análisis de los datos en el cual se describieron las características de la muestra y se establecieron las relaciones entre las variables del estudio. Para los resultados se realizó el proceso estadístico mediante análisis de ecuaciones estructurales.

Cabe notar que ya que se pretendía identificar el riesgo de TCA, se consideran puntos de corte más sensibles o menores a los definidos para detectar condiciones clínicas en la población, para ello se retoma el límite sub clínico propuesto para Colombia en cada uno de los Factores y en la puntuación total del EAT.

\section{Análisis de datos}

Para el análisis de los datos se tuvieron en cuenta los siete pasos consecutivos que permiten alcanzar el análisis estructural del Modelo de Ecuaciones Estructurales MEE:

Paso 1. Desarrollo del Modelo Hipotético Inicial Basado en una teoría y hallazgos empiricos.- El desarrollo del modelo implica la ubicación de las variables de acuerdo con relaciones de asociación propuestas teóricamente; en este proceso es necesario tener en cuenta el error de especificación, ya que aunque se analizan relaciones causales entre variables, siempre pueden hacerse especulaciones debido a la contigüidad en tiempo y espacio de las mismas, llegando a conclusiones erróneas; puede presentarse la omisión de variables importantes o de otras variables relacionadas que afecten la toma de medidas estimadas para el modelo.

Paso 2. Construcción de un Diagrama de Relaciones.- Se expresaron las relaciones de asociación a través de un diagrama guía que muestra las conexiones entre los conceptos (variables); se introdujo el constructo que sostiene a los conceptos y se aclaró teóricamente la definición de las relaciones. Los diagramas representan todas las relaciones, así mismo la teoría los justifica. En este paso se clarificaron dos tipos de constructos; los exógenos, conocidos como variables independientes que no son asociadas o predichas a través de otras variables en el modelo; $y$, los endógenos que pueden ser predichos por una o más variables y se conocen también como variables dependientes.

Paso 3. Toma de las Medidas de las Variables Observadas.- Se especificaron las medidas de las variables del constructo para medir el modelo; es aquí en donde se efectuó la medición de las variables que sostienen el constructo, utilizando pruebas claramente establecidas psicométricamente o diseñándolas 
en caso de ser necesario. Posteriormente se trasladó el diagrama a ecuaciones estructurales en donde cada constructo endógeno se toma como una variable dependiente en una ecuación por separado. Se calculó el coeficiente estructural incluyendo el término de error posible para cada ecuación (suma de errores de especificación). Finalmente se generaron correlaciones entre constructos e indicadores (variables) evaluando su significancia estadística.

Paso 4. Estimación del Modelo Propuesto.- Se analizó la varianza y covarianza de cada variable a través de matrices; comparando diversas muestras poblacionales para así entender el patrón de relaciones del constructo. Se usó la correlación producto momento de Pearson para el análisis multivariado. La covarianza y la correlación permiten observar las relaciones causales entre las variables estudiadas y así mismo evidenciar la estructura adecuada del modelo que integra dichas variables.

Paso 5. Evaluación de la Identificación del Modelo.- En este paso el trabajo se concentró en evidenciar los posibles problemas del modelo; requirió una revisión detallada de la concordancia entre los resultados obtenidos a partir del MEE y la teoría que sustenta al modelo.

Paso 6. Evaluación de resultados y ajuste.- En este momento se evalúa si los resultados y el modelo propuesto pueden ser asumidos como un MEE, para ello se lleva a cabo primero una evaluación global del modelo y, posterior a ello, una por separado.

Se buscan los elementos que muestren inadecuación del modelo, teniendo en cuenta la existencia de errores de va- rianza negativos para algún constructo, coeficientes de correlación que exceden los límites estandarizados, error estándar muy extenso; todo para observar su efecto en los resultados y corregir el problema existente.

\section{Resultados}

La muestra estaba conformada por 417 personas entre los 16 y 25 de los cuales la mayor parte eran mujeres (70.5\%), cuya procedencia estaba equilibrada entre Bogotá e Ibagué (Tabla 1).

Tabla 1. Descripción sociodemográfica de los participantes.

\begin{tabular}{|c|c|c|c|c|}
\hline Variable & $\%$ & Variable & $M$ & $D T$ \\
\hline Género & & Antecedentes Psiquiátricos & & \\
\hline Hombre & 12329.5 & $\mathrm{Si}$ & 44 & 10.6 \\
\hline Mujer & 29470.5 & No & 373 & 89.4 \\
\hline Procedencia & & Edad & 20.02 & 3.53 \\
\hline Ibagué & 24558.8 & Rango & $16 \mathrm{a}$ & 25 \\
\hline Bogotá & 17241.2 & & & \\
\hline
\end{tabular}

$\mathrm{Al}$ medir la talla de los participantes se encontró que la estatura promedio era de $1.65 \mathrm{~m}$, con un límite mínimo de $1.46 \mathrm{~m}$ y un máximo de $1.90 \mathrm{~m}$; respecto del peso se encontró que en promedio las personas incluidas en el estudio pesaban $59.86 \mathrm{~kg}$, con un límite mínimo de $41 \mathrm{~kg}$ y uno máximo de $111 \mathrm{~kg}$. En la medida del IMC se discriminaron por género los promedios con 21.55 para las mujeres y 22.19 para los hombres. De otra parte, el 65\% de los participantes tenía un peso normal y al menos el 35\% de la población presentó problemas de peso, ya fuera por déficit o por exceso (Tabla 2).

Tabla 2. Descripción de talla, peso e índice de masa corporal Niveles del EAT y de Depresión de los participantes.

\begin{tabular}{llllllll}
\hline Variables & $M$ & $D T$ & Min & Max & Variable & $f$ & $\%$ \\
\hline Talla & 1.65 & 0.08 & 1.46 & 1.90 & Nivel de peso & & \\
Peso & 59.86 & 11.85 & 41 & 111 & Bajo & 66 & 15.8 \\
IMC & & & & & Normal & 271 & 65.0 \\
Mujeres & 21.55 & 3.37 & & & Sobrepeso & \\
Hombres & 22.19 & 3.71 & & & Obesidad & 54 & 12.9 \\
& & & & & 12 & 2.9 \\
Niveles del EAT & $f$ & Percentiles & & Niveles & $f$ & Percentiles \\
Normal & 153 & 36.8 & & Sin depresión & 388 & 93.3 \\
Riesgo moderado & 137 & 32.9 & & Con síntomas depresivos & 21 & 5.0 \\
Alto riesgo & 104 & 25.0 & & Depresión leve & 6 & 1.4 \\
Población clínica & 21 & 5.0 & & Depresión mayor & 1 & 0.2 \\
\hline
\end{tabular}

Se encontró que al menos el 63\% de los participantes tenía algún riesgo de padecer un TCA, entre ellos 32.9\% riesgo moderado, $25 \%$ alto riesgo y un $5 \%$ podría considerarse población clínica. Cabe anotar que el 33\% tenía indicadores de riesgo. De otra parte, en una alta proporción de la muestra los puntajes denotaban ausencia de depresión y solo el 6.6\% presentaba síntomas depresivos específicos o se encontraba deprimido, con un único caso de depresión mayor (Tabla 3). Al realizar la comparación por género y por procedencia de los puntajes obtenidos en depresión no se encuentra diferencia significativa al respecto. 
Tabla 3. Descripción de las variables predictoras en relación con el género y la procedencia de los participantes.

\begin{tabular}{|c|c|c|c|c|c|c|c|c|}
\hline \multirow[t]{2}{*}{ Variables } & \multicolumn{2}{|c|}{ Mujeres } & \multicolumn{2}{|c|}{ Hombres } & \multicolumn{2}{|c|}{ Ibagué } & \multicolumn{2}{|c|}{ Bogotá } \\
\hline & $M$ & $D T$ & $M$ & $D T$ & $M$ & DT & $M$ & $D T$ \\
\hline Optimismo & 15.66 & 3.18 & 14.96 & 3.31 & 15.68 & 2.98 & 15.13 & 3.54 \\
\hline Sesgo opt. & 3.49 & 1.83 & 4.34 & 1.99 & 3.61 & 1.88 & 3.93 & 1.97 \\
\hline Pesimismo & 2.54 & 2.11 & 2.41 & 2.05 & 2.50 & 2.04 & 2.51 & 2.18 \\
\hline Depresión & 10.66 & 6.9 & 10.54 & 6.9 & 11.00 & 7.09 & 10.09 & 6.31 \\
\hline Afront Evitativo & 1.22 & 0.44 & 1.19 & 0.42 & 1.20 & 0.43 & 1.22 & 0.43 \\
\hline Afront C. Problema & 1.53 & 0.43 & 1.45 & 0.37 & 1.48 & 0.40 & 1.53 & 0.44 \\
\hline
\end{tabular}

La población en general obtuvo puntajes altos en la escala que medía optimismo (15.66), con diferencias importantes referidas al género y la procedencia, ya que las mujeres pun- tuaron más alto en optimismo que los hombres y los Ibaguereños obtuvieron puntajes más altos que los Bogotanos (Tabla 4).

Tabla 4. Medias EAT por género, ciudad y el Baremo Colombia.

\begin{tabular}{|c|c|c|c|c|c|c|c|c|c|c|c|c|}
\hline \multirow[b]{2}{*}{ EAT } & \multicolumn{2}{|c|}{ Mujeres } & \multicolumn{2}{|c|}{ Baremo Colombia } & \multicolumn{2}{|c|}{ Hombres } & \multicolumn{2}{|c|}{ Baremo Colombia } & \multicolumn{2}{|c|}{ Ibagué } & \multicolumn{2}{|c|}{ Bogotá } \\
\hline & $M$ & $D T$ & $M$ & $D T$ & $M$ & $D T$ & $M$ & $D T$ & $M$ & $D T$ & $M$ & $D T$ \\
\hline Total & 45.05 & 12.19 & 49.57 & 14.45 & 39.93 & 8.36 & 43.77 & 11.72 & 44.40 & 11.67 & 42.31 & 11.00 \\
\hline Factor 1 & 15.58 & 4,37 & 17.04 & 6.08 & 13.91 & 3.40 & 13.89 & 5.18 & 15.26 & 4.17 & 14.84 & 4.18 \\
\hline Factor 2 & 8.04 & 3.84 & 9.60 & 3.90 & 6.04 & 2.69 & 8.42 & 3.44 & 7.73 & 3.71 & 7.05 & 3.54 \\
\hline Factor 3 & 9.65 & 3.50 & 10.17 & 3.88 & 9.38 & 2.95 & 9.88 & 3.13 & 9.77 & 3.32 & 9.28 & 3.38 \\
\hline Factor 4 & 5.70 & 2.22 & 6.39 & 2.78 & 5.04 & 1.47 & 6.16 & 2.50 & 5.55 & 2.10 & 5.43 & 1.98 \\
\hline Factor 5 & 6.05 & 3.25 & 6.37 & 3.18 & 5.55 & 2.92 & 5.43 & 2.52 & 6.04 & 3.41 & 5.69 & 2.77 \\
\hline
\end{tabular}

Factor 1: Preocupación por la ganancia de peso corporal. Factor 2: Preocupación por la cantidad de calorías contenidas en los alimentos. Factor 3: Preocupación obsesiva por la comida. Factor 4: Bulimia. Factor 5: Preocupación social por la ganancia de peso.

Las mujeres (45.05) obtuvieron mayores puntajes totales en el EAT que los hombres (39.93) (Tabla 4). Al comparar los puntajes obtenidos en la escala total del EAT y por factores se encontró la existencia de diferencias significativas respecto del género y la procedencia. En los puntajes de depresión obtenidos comparados por género y procedencia no se evidencian diferencias significativas (Tabla 5).

Tabla 5. Comparación por género y procedencia de las variables predictoras de EAT y del riesgo de TCA.

\begin{tabular}{|c|c|c|c|c|c|}
\hline \multicolumn{6}{|l|}{ Comparación por género } \\
\hline & Suma de Cuadrados & $d f$ & Media Cuadrática & $F$ & $p$ \\
\hline EAT & 3056.67 & 1 & 3056.67 & 19.22 & .00 \\
\hline Factor 1 & 242.16 & 1 & 242.16 & 14.30 & .00 \\
\hline Factor 2 & 345.29 & 1 & 345.29 & 27.49 & .00 \\
\hline Factor 3 & 6.52 & 1 & 6.52 & 0.58 & .44 \\
\hline Factor 4 & 37.77 & 1 & 37.77 & 9.15 & .00 \\
\hline Factor 5 & 21.51 & 1 & 21.51 & 2.14 & .14 \\
\hline Depresión & 4.87 & 1 & 4.87 & 0.31 & .57 \\
\hline Satisfacción Imagen Corporal & 34.28 & 1 & 34.28 & 14.79 & .00 \\
\hline \multicolumn{6}{|l|}{ Comparación por procedencia } \\
\hline & Suma de Cuadrados & $d f$ & Media Cuadrática & $F$ & $p$ \\
\hline EAT & 795.97 & 1 & 795.97 & 4.84 & .02 \\
\hline Factor 1 & 17.67 & 1 & 17.67 & 1.01 & .31 \\
\hline Factor 2 & 46.25 & 1 & 46.25 & 3.48 & .06 \\
\hline Factor 3 & 24.73 & 1 & 24.73 & 2.20 & .13 \\
\hline Factor 4 & 1.68 & 1 & 1.68 & .39 & .52 \\
\hline Factor 5 & 12.46 & 1 & 12.46 & 1.24 & .26 \\
\hline Depresión & 25.53 & 1 & 25.53 & 1.64 & .20 \\
\hline
\end{tabular}

Factor 1: Preocupación por la ganancia de peso corporal. Factor 2: Preocupación por la cantidad de calorías contenidas en los alimentos. Factor 3: Preocupación obsesiva por la comida. Factor 4: Bulimia. Factor 5: Preocupación social por la ganancia de peso. 
Respecto a cada factor del EAT se observa que las mujeres puntuaron más alto en cuatro de los cinco factores evaluados (Factor $1=15.58$, Factor $2=8.04$, Factor $4=5.70$, Factor $5=6.05)$ y obtuvieron puntajes similares a los de los hombres en el factor 3 correspondiente a la preocupación obsesiva por la comida. En cuanto a las dos ciudades, los resultados del EAT total y por factor muestran que los Ibaguereños obtuvieron calificaciones por encima de las obtenidas por los bogotanos, y más cercanos al punto de corte clínico indicado para Colombia. Ya que se retoman calificaciones sub clínicas cabe notar que las medias en ambos casos muestran puntajes de riesgo elevados en todos los factores con desviaciones estándar similares a las de la población clínica (Tabla 5).

\section{Modelo Predictivo}

En la figura 1 se presenta el modelo predictivo del Riesgo de Padecer Trastorno de la Conducta Alimentaria, allí se muestran el total de varianza explicado del Riesgo por la interacción de las variables y el peso de varianza explicado por cada una de ellas. Se evidencia una relación predictiva entre los antecedentes psicológicos familiares (padre y madre), el deseo de disminución del peso, la satisfacción con la imagen corporal, el estilo de afrontamiento evitativo o centrado en la emoción y la depresión, y el riesgo de padecer un trastorno de la conducta alimentaria.

La varianza total explicada fue del $34 \%$, considerada aceptable en estudios de ciencias sociales. En orden jerárquico las variables que más peso de varianza aportaron al modelo son: depresión (32\%), antecedentes psicológicos familiares $(22 \%)$, estilo de afrontamiento evitativo (14\%), satisfacción con la imagen corporal (12\%), deseo de disminuir el peso $(11 \%)$ y género $(-16 \%)$.

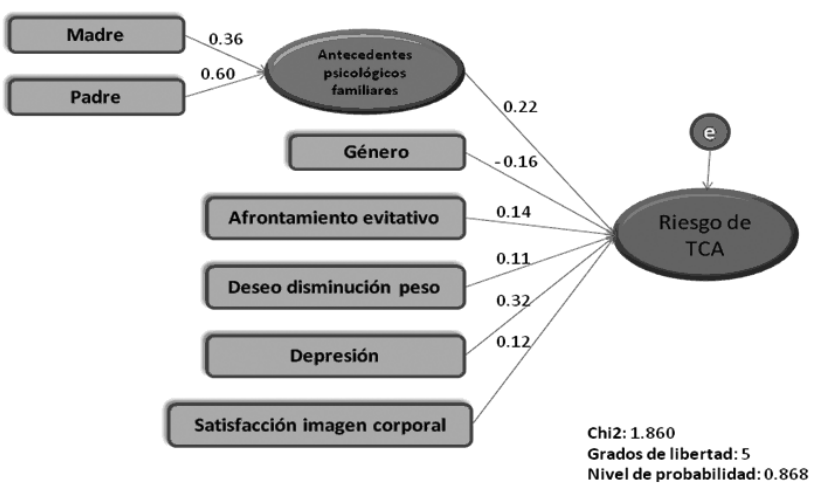

Figura 1. Modelo Predictivo del Riesgo de Padecer Trastorno de Conducta Alimentaria.

De acuerdo con los distintos índices considerados para definir el nivel de ajuste del modelo, el Chi2 calculado para el modelo y los $g l$ (5) y un índice de bondad de ajuste de 1.2 se puede afirmar que está identificado y ajustado a los datos obtenidos, esto quiere decir que se acepta parcialmente la hipótesis de trabajo ya que antecedentes psicológicos familiares, género, deseo de disminución del peso, satisfacción con la imagen corporal, estilo de afrontamiento evitativo y depresión en conjunto predicen el riesgo de padecer un TCA; y que variables como la salud percibida y el IMC no estaban incluidas en el modelo.

\section{Discusión y Conclusiones}

Existen pocos estudios que aclaren el efecto de algunos factores psicosociales sobre la aparición y curso de los Trastornos de Conducta Alimentaria, por tanto los resultados de la presente investigación pueden orientar la dirección de futuros estudios en el tema y facilitar el desarrollo de acciones de prevención y tratamiento de estos trastornos. Incluso, algunos de los factores pueden ser vistos bajo una perspectiva positiva y pensar más allá del riesgo abordando el problema desde una mirada protectiva.

La muestra estaba conformada por adolescentes y jóvenes que procedían de dos ciudades, con estatura promedio típica del género. Al igual que otras poblaciones, una parte de la muestra reportó tener antecedentes psiquiátricos; esto ratifica la idea de que sin lugar a dudas es importante estudiar la salud de los adolescentes ya que los trastornos mentales aparecen cada vez a más temprana edad y es precisamente la adolescencia el momento crítico de inicio de síntomas de casi todos los trastornos mentales mayores (Alzate, 2003). Cabe anotar que para el caso de los sujetos estudiados la prevalencia reportada de trastornos psiquiátricos fue menor a la reportada en el Estudio Nacional de Salud Mental en Colombia (MEN, 2003), que mostró una prevalencia en el último año de $16 \%$. Igualmente dicho estudio mostro diferencias significativas en el tipo de trastorno reportado por hombres y mujeres, corroborando los hallazgos obtenidos al respecto en el presente estudio, ya que se evidencia mayor prevalencia de TCA en mujeres que en hombres.

En cuanto al peso de la muestra, se presentan las diferencias normales entre hombres y mujeres y se encuentra que una buena parte de la población tenía problemas de exceso de peso, en su mayoría de sobrepeso, aunque algunos casos de obesidad pre mórbida fueron registrados; no obstante, esta población parece tener mayores dificultades al respecto que las incluidas en estudios como el de Gamboa, López y Quintero (2007). En ésta línea, la Encuesta Nacional de Alimentación y Nutrición (Organización Mundial de la Salud OMS, 2005) mostró que el problema del sobrepeso y obesidad en niños y adolescentes del mundo ha ido en aumento, especialmente en países en vía de desarrollo como Colombia, en la que se encontró que al menos el 10\% de adolescentes presentaban sobrepeso. Esto resulta preocupante pues las personas con sobrepeso durante la adolescencia tienden a mantener dicha condición el resto de su vida, con las implicaciones de salud que conlleva: deficiencias cardiacas, enfermedades metabólicas, disfunciones respiratorias y anomalías en el sistema digestivo, entre otras (Gamboa et al., 2007). 


\section{Modelo predictivo de riesgo de padecer TCA}

Se acepta parcialmente la hipótesis de trabajo ya que antecedentes psicológicos familiares, género, deseo de disminución del peso, satisfacción con la imagen corporal, el estilo de afrontamiento evitativo y la depresión en conjunto predicen el riesgo de padecer un TCA, pero variables como la salud percibida y el IMC no se incluyen en el modelo.

Con relación al riesgo reportado de padecer un TCA, en la misma línea que estudios como los de Azevedo et al. (2010), Lugli \& Vivas (2006), Waller \& Sheffield (2008) y esta investigación muestra que los adolescentes presentan un mayor riesgo de desarrollar algún TCA al parecer debido a las preferencias sociales por la delgadez y a un estilo de vida centrado en la imagen y la figura, propios de esta etapa de la vida. De igual forma, los medios de comunicación y los agentes sociales influyen en la difusión de un ideal estético de delgadez y han estimulado que los valores positivos de las personas estén asociados cada vez más a la imagen, especialmente en los jóvenes, quienes se ven presionados a alcanzar dichos estándares, pues predominan valores centrados en la deseabilidad social y la pertenencia a grupos que proporcionan estatus (Engler et al., 2006; Lugli \& Vivas, 2006; Rivarola, 2003; Rivarola \& Penna, 2006). Al igual que otros estudios (Buckner, Schmidt, Silgado \& Timpano, 2010; Castro, 2002; Sepúlveda et al., 2001; Steiger, 2007); la presente investigación revela la existencia de una tendencia femenina a desarrollar TCA por encima de los hombres: ya se había planteado una proporción de prevalencia de estos trastornos de 9 casos femeninos por cada caso masculino. Más que el rol biológico que cumplen las mujeres se encuentra un cambio en las exigencias sociales sobre el éxito y la apariencia física para ambos géneros; así el hecho de ser mujer no está por encima de la importancia relativa dada a la apariencia y a ajustarse a los parámetros impuestos por la familia y la sociedad. Es decir que hombres y mujeres que conceden alta valencia a la imagen corporal que cumple los parámetros sociales tendrían mayor riesgo de padecer un TCA y esto no se daría por el simple hecho de ser mujer.

Aunque persiste el interrogante de porqué las mujeres tienen mayor riesgo de padecer un TCA, han surgido algunas explicaciones que parten del hecho de que el estereotipo de figura corporal que promueve la sociedad tiene mayores exigencias para las mujeres que para los hombres, ya que la delgadez es altamente valorada social, laboral y profesionalmente. Exigencias que llevan a las mujeres a comportarse de manera extrema incluso en contra de la propia salud para obtener la delgadez deseada; adicionalmente, las mujeres reciben mayor presión por su propia apariencia (tamaño de los senos y caderas, estrechez de la cintura, sinuosidad del cuerpo, calidad del cabello y la piel) y los hombres enfatizan más en los aspectos instrumentales del cuerpo de la mujer (cierta figura se asocia a mayor receptividad sexual y de progenie); lo que ha llevado a que se instaure cada vez más un discurso nutricionista más aceptado entre las mujeres, pero que sin duda toca a los hombres (Mercado, 2008; Giovazolias y Koskina, 2010).

La historia familiar psiquiátrica en los dos progenitores resultó estar estrechamente asociada al riesgo de TCA; ya otros estudios habían mostrado como la historia de patologías o alteraciones de personalidad en los padres conllevan mal pronóstico en el riesgo de padecer un TCA y juegan un papel etiológico en estos trastornos (Bardone \& Fitzsimmonsa, 2011; Beato-Fernández \& Rodríguez Cano, 2005; Lara \& Akiskal, 2006; Ochoa de Alda, Espina \& Ortego, 2006). De igual forma que el presente estudio, los hallazgos de Palmer (2008) y el de Reba-Harrelson, Von Holle, Hamer, Torgersen, Reichborn-Kjennerud \& Bulik, (2010); Kluck (2010) reportan que los diagnósticos de Trastorno de Conducta Alimentaria son más frecuentes en jóvenes cuyas madres han presentado un TCA.

En este grupo de jóvenes en general los índices de depresión y de síntomas depresivos son menores que los hallados en otros estudios de prevalencia realizados en población similar que arrojaron índices de depresión en jóvenes entre el 8\% y el 30\% (Arrivillaga, Cortés, Goicochea \& Lozano, 2003). No obstante, no se hallaron diferencias significativas entre hombres y mujeres en el reporte de síntomas depresivos, contrario a los hallazgos de la Encuesta Nacional de Salud Mental (MEN, 2003) en Colombia y en otros estudios (Pardo, Sandoval, \& Umbarila, 2004; Restrepo, 1997; Unikel \& Gómez-Peresmitré, 2004); en los que se muestra que las mujeres tienden a padecer con mayor frecuencia trastornos depresivos, o por lo menos los reportan con mayor frecuencia que los hombres o son diagnosticadas más frecuentemente debido a ciertas dificultades existentes en el sistema nosológico relacionadas con los síntomas por género (Álvarez \& Londoño, 2011).

Lo anterior podría explicarse si se tiene en cuenta que la muestra estaba conformada predominantemente por mujeres y esto pudo afectar los resultados obtenidos al respecto; no obstante, es imposible obviar el cambio del perfil epidemiológico que el mundo está sufriendo, de aquí que algunos trastornos como los TCA y la depresión ya no sean exclusivamente femeninos. De hecho, el US Department of Health and Human Services y la National Institute of Mental Health (2005) plantean que además de ser cada vez más frecuente la depresión en hombres, los casos no son debidamente detectados debido a que los hombres sienten la depresión de manera diferente; contrario a las mujeres, los síntomas más frecuentes son fatiga, irritabilidad, pérdida de interés en el estudio o pasatiempos favoritos, problemas de sueño, abuso en el consumo de SPA; y que éstos, antes que reconocer los síntomas, pedir ayuda o solicitar el tratamiento adecuado, prefieren mantener sus síntomas en el anonimato. No obstante, es preciso desarrollar más investigación al respecto pues el tema trasciende del todo los alcances de la presente investigación.

Sin embargo, es preciso anotar que en el presente estudio, contrario a los resultados obtenidos en la Encuesta de Salud Mental en Colombia (MEN, 2003), no se evidencian 
diferencias con respecto a la prevalencia de depresión entre Ibagué y Bogotá. Pero estos resultados deben ser tomados con prudencia, ya que un número reducido de jóvenes obtuvo puntajes altos en depresión y aunque la muestra pretendió ser representativa, el hecho de que se haya autoseleccionado los participantes limita la generalización de los resultados.

$\mathrm{Al}$ igual que en esta investigación, los TCA han sido patologías relacionadas con la depresión; de hecho, estudios sobre marcadores biológicos han demostrado una sorprendente similitud entre depresión y los TCA (Chinchilla, 2005); sin embargo, aún a pesar de la asociación encontrada en este estudio entre estos factores, la naturaleza de dicha asociación es controvertida debido a la comorbilidad frecuente y al hecho de que éste y otros estudios han sido de corte trasversal. Adicionalmente, es preciso anotar que aunque que si bien la mayor parte de la población tiene algún nivel de riesgo de padecer TCA y éste parece estar asociado con la depresión, un grupo numeroso de ellos no tienen síntomas depresivos importantes; por ello no es posible concluir que siempre que hay alto riesgo de TCA exista depresión y viceversa. Podría pensarse que la depresión es un factor que puede agudizar algunos síntomas de TCA como: baja autoestima, aislamiento social, distorsión de la imagen corporal; y a su vez, los TCA pueden tener un efecto similar sobre la depresión en los mismos síntomas.

Los jóvenes incluidos en el estudio se preocupan intensamente por ganar peso, por la cantidad de calorías contenidas en los alimentos y por la comida en general, con una marcada predominancia en las mujeres. Esto confirma los resultados obtenidos en otras investigaciones que muestran que las mujeres, en comparación con hombres de la misma edad y nivel de estudios, se preocupan más por la ingesta de alimentos, la comida y frecuentemente desean ser más delgadas o perder peso, independientemente de que sea necesario (Acosta et al., 2005; Gómez-Peresmitré \& Acosta, 2002; Peláez, Labrador \& Raich, 2005); se muestran insatisfechos con su imagen corporal, les gustaría parecerse a modelos publicitarias o no están de acuerdo con la talla de pantalón que usan (Mercado, 2008).

Desde el punto de vista sociocultural, se ha argumentado una creciente presión social sobre el peso y la imagen corporal, que se traduce en una propensión a seguir ciertas pautas alimentarias restrictivas, irreales e insanas. En Canadá, los estudios reportan que el $70 \%$ de todas las mujeres con sobrepeso, peso normal o delgadas, deseaban reducir el mismo (Millar, 1991). Los resultados obtenidos en este estudio, en el que la deseabilidad del cambio de peso puntuó una media de 1,18 para las mujeres y de ,90 para los hombres, coinciden con los de diversos estudios realizados, en los que se observó que un gran número de mujeres quisieran pesar menos aunque posean un peso acorde a su edad y talla (Gómez-Peresmitré \& Acosta, 2003); explicar el hecho de que en el modelo no esté incluido el IMC que representa la relación talla - peso real y resulta ser independiente de la satisfacción con la imagen corporal en esta población.
La insatisfacción con la imagen corporal, las dietas y las ideas sobrevaloradas de un cuerpo delgado son factores prevalentes en la mayoría de las adolescentes; sumadas a la presión social publicitaria que presentan modelos que fomentan la delgadez como símbolo de éxito en la vida (Rivarola \& Penna, 2006), todas relacionadas con el hecho de padecer un TCA. La satisfacción con la imagen corporal, considerada una variable moderadora del desarrollo de trastornos de la conducta alimentaria, debe ser abordada desde dos puntos de vista: 1) el relacionado con la influencia del modelo estético corporal actualmente predominante, que se manifiesta a través de creencias y actitudes hacia la obesidad, y 2) el relacionado con la discrepancia entre una figura real e ideal, que genera insatisfacción con la totalidad del cuerpo o con partes del mismo (Unikel \& Gómez-Peresmitré, 2004). Resultados como los hallados por Adab, Duda, Hiam \& Pallan (2011), Çatikkas, 2011 y Sepúlveda, Botella \& León (2001), ponen de manifiesto que las medidas actitudinales de la imagen corporal han originado mayores tamaños del efecto que las medidas perceptivas, por lo tanto se puede pensar que las actitudes y las creencias sobre uno mismo son las que muestran asociación más estrecha con la insatisfacción con la imagen corporal.

Sin duda el deseo de cambio de peso, evidente en la población estudiada y en otras poblaciones similares, es resultado de la insatisfacción con la imagen corporal; ya algunos estudios han reportado cómo un gran número de adolescentes, con y sin TCA, presentan alta preocupación por la imagen corporal e insatisfacción con su forma corporal. Esto ha sido relacionado con el hecho de que la adolescencia es una etapa evolutiva de crisis, en la que tienen lugar cambios físicos y psicológicos significativos, y factores interpersonales y familiares que influyen en la formación y aceptación de la imagen corporal adquieren gran relevancia en la escala de valores del joven (Edlund, Gustafsson, Kjellin \& Norring, 2009; Giovazolias \& Koskina, 2010; Rivarola, 2003).

Esta carrera por alcanzar la imagen perfecta, ha sido descrita por Welch, Miller, Ghaderi \& Vaillancourt (2009) y por Watt \& Ricciardelli, (2012) como una marcada tendencia al perfeccionismo que pone al individuo en una carrera por emular arquetipos inalcanzables que proponen extremos de perfección, ya que al comparar su figura con "el cuerpo deseado" se genera una profunda insatisfacción con la imagen corporal y se da paso al inicio y mantenimiento de los TCA. Este perfeccionismo es orientado en dos sentidos: 1) Externamente: dirigido a satisfacer a los otros, e 2) Internamente: centrado en el uso permanente de la crítica de sí mismos; combinación que hace que los estándares de exigencia sean muy altos y que las mujeres dirijan todo el tiempo sus esfuerzos a alcanzar la perfección, llevándolas a realizar dietas e incluso a someterse a tortuosos procedimientos médicos o estéticos, por encima del propio bienestar físico y emocional.

En esta misma línea las mujeres presentan mayor preocupación social por la ganancia de peso que los hombres, y más las procedentes de Ibagué que las de Bogotá. Estos resultados pueden estar asociados al hecho de que estas ciuda- 
des tienen climas diferentes; el clima cálido de Ibagué hace que las prendas de vestir usadas principalmente sean de colores claros, trasparentes, vaporosas, escotadas y ajustadas, características que demarcan más la figura poniendo en evidencia, ante los demás, los atributos del cuerpo; por el contrario en Bogotá se usan prendas de vestir mas abrigadas y de colores oscuros que ocultan la figura y dan oportunidad de usar prendas intimas reductoras.

Por otra parte estos resultados también se pueden deber a las características culturales de las ciudades y de las regiones en las que se encuentran (Acosta et al. 2005), así como a su tamaño y a los hábitos (Anderson, Simmons, Martens, Ferrier, \& Sheehy, 2006) y pautas de socialización (Crow, Eisenberg, Story, \& Neumark-Sztainer, 2006). Sin duda es importante estudiar más a fondo este aspecto teniendo muestras equilibradas por regiones que permita corroborar estos datos y hacerlos concluyentes.

Los jóvenes de la muestra, independientemente del género, mostraron tendencia a usar estrategias de afrontamiento evitativo, hallazgos coincidentes con investigaciones previas que han demostrado que los adolescentes al enfrentarse a una situación estresante suelen usar estilos de afrontamiento centrados en la emoción, que pueden variar según el sexo y fortalecerse con la edad (Garnefski \& Kraaij; Tusaie \& Patterson, 2006; Hampel \& Petermann, 2006). Por el contrario, reportes de anteriores estudios evidencian la existencia de diferencias particulares entre hombres y mujeres en el afrontamiento evitativo o centrado en la emoción, las investigaciones de Engler et al. (2006); Grylli, Wagnera, HafferlGattermayera, Schoberb y Karwautz (2005) y Renk \& Creasey (2003) señalan que el estilo de afrontamiento centrado en la emoción es más propio de las mujeres que de los hombres.

Durante la adolescencia, el afrontamiento del estrés está mediado por la internalización de la nueva imagen corporal, la búsqueda de independencia y autonomía, la identificación y la individualización; la incorporación de un sistema de valores; la perspectiva de futuro y el desarrollo de una identidad psicosexual. Aún así, el desarrollo de un estilo propio de afrontamiento, está influenciado por los patrones de socialización a los que están expuestos, y provienen principalmente de los modelos familiares de respuesta ante el estrés (Solís \& Vidal, 2006). Es decir, que se espera que los padres de los jóvenes incluidos en la muestra también muestren una clara tendencia a presentar conductas evitativas ante las situaciones problema.

La adquisición de un estilo de afrontamiento de jóvenes en edad escolar está relacionada con las características del estilo de paternidad y la familia. Así, aunque el vínculo con los padres sea pobre, estas figuras contribuyen de manera significativa e independiente, en el aprendizaje de estrategias específicas de afrontamiento (Pardo et al. 2004). Podría pensarse que un estilo de paternidad más autoritario, basado en la coerción y el castigo, llega a generar en los jóvenes comportamientos de sumisión o de indefensión, y la evitación futura de situaciones que representan problema o dificultad.
Por otro lado si en la familia se promueve y prioriza el aprendizaje de competencias académicas, por encima del aprendizaje de competencias para la vida, el joven se forma sin herramientas básicas para el afrontamiento de situaciones cotidianas de manera autónoma y eficiente, y estará dependiendo de lo que otros propongan a la hora de resolverlas.

Así mismo, el grupo de pares se constituye en un importante "modelo de referencia" en la adquisición de estrategias de afrontamiento, una vez que brinda la posibilidad de tomar decisiones, de tener poder y seguridad, proporciona prestigio y libertad, en el cual existen emociones, ideas, ambiciones y expectativas, y se desarrollan habilidades que con frecuencia resultan insuficientes para el establecimiento de interacciones sociales satisfactorias y el adecuado afrontamiento de las situaciones problemáticas (Ouwehand, De Ridder \& Bensing, 2008). En esa medida, si los jóvenes cuentan con amigos y compañeros con escasas e ineficientes estrategias de afrontamiento, ellos adquirirán patrones de respuesta ante las situaciones, que evalúan como amenazantes, igualmente negativos y que los distancian de la solución efectiva de estas situaciones. Sin duda este resulta ser otro tópico de investigación de gran importancia que debe ser abordado en próximas investigaciones.

Sin lugar a dudas, resulta necesario continuar en la búsqueda del conocimiento de las estrategias y estilos de afrontamiento en los adolescentes ya que esto permitiría comprender e intervenir mejor sobre la problemática social que presentan los jóvenes. Adicionalmente, en la medida que se logre hacer que los adolescentes afronten de mejor forma ciertas situaciones se logrará mayor adaptación y por ende mayor bienestar para esta población.

En conclusión, no es la primera vez que estudios sobre los TCA contemplan variables como las relacionadas en este estudio. Lo que hace diferentes y significativos los resultados que aquí se presentan y discuten, es la propuesta de un modelo multifactorial para la explicación de estos trastornos, no un modelo de tipo lineal sino uno de modulación, en donde las variables se modulan unas a otras. En todo caso, el Modelo Predictivo de Riesgo de Padecer Trastorno de Conducta Alimentaria, que se describe como resultado de éste estudio, es sólo una propuesta en el avance de la comprensión de estos trastornos, por lo que requiere ser replicada y analizada en diferentes grupos poblacionales.

Por otro lado, pautas de crianza y prácticas de alimentación inadecuadas por parte de las madres con TCA, influyen en los hábitos de alimentación de los hijos, llevándolos a un consumo restrictivo de alimentos o a la mala alimentación y a posteriores problemas con el peso; en conjunto con una tendencia genética de depresión y/o de TCA, y una insatisfacción con la imagen corporal, donde las dificultades para relacionarse con otros o los conflictos con los padres, entre otros, se atribuyen a la figura o la apariencia física, y ante la no identificación de estrategias que permitan afrontar y solucionar la inconformidad, el joven se aísla, evita la interacción social, pierde interés por sus actividades cotidianas, llegando a estados de depresión y busca obsesivamente reducir su pe- 
so y mejorar su imagen, sometiéndose a cualquier estrategia, aun a aquellas que representan una amenaza para la salud, o el desarrollo de un trastorno de la conducta alimentaria.

\section{Limitaciones y futuras direcciones}

Lamentablemente pocos investigadores se han volcado a esta área con la intención de identificar un Modelo de Predicción del Riesgo de Padecer TCA, por lo que sólo podemos hablar de resultados preliminares. En consecuencia, futuras investigaciones deberán replicar estos hallazgos con población clínica para poder considerarlos como concluyentes.

Entre las principales limitaciones del presente estudio se encuentra: la mayor participación de mujeres, la predominancia de personas de una de las ciudades y que la muestra haya sido autoseleccionada. Por tanto, es preciso replicar la investigación con muestras procedentes de diferentes regiones, que incluyan población clínica y equilibren mejor el género. No obstante, cabe anotar que estas son características difíciles de controlar en un medio en el que los recursos de investigación no son abundantes. De otra parte el hecho

\section{Referencias}

Adab, P., Duda, J. L., Hiam, L. C., \& Pallan, M. J. (2011). Body image, body dissatisfaction and weight status in south asian children: a crosssectional study. Public Healt, 11(21): 2-8. Doi:10.1186/1471-2458-11-21

Acosta, M. V., Llopis, J. M., Gómez, G., \& Pineda, G. (2005). Evaluación de la conducta alimentaria de riesgo. Estudio transcultural entre adolescentes de España y México. International Journal of Psychology and Psychological Therapy, 5, 223-232. Recuperado de http:/ http://www.dialnet.unirioja.es/servlet/articulo?codigo=1415698.

Alzate, M. (2003). Epidemiología Psiquiátrica, Revista Colombiana de Psiquiatria, $32 \quad$ (1) 87-94. Recuperado de http://www.scielo.org.co/scielo.php?script=sci_arttext\&pid=S0034745 02003000100006\&lng $=$ en\&nrm $=$ iso $>$. ISSN 0034-7450.

Álvarez, N., \& Londoño, C. (2011). ¿Se deprimen los hombres? ¿Diferente de las mujeres? España: Editorial Académica Española.

American Psychiatric Association (APA). (2000). Diagnostic and Statistical Manual of Mental Disorders: DSM-IV-TR. ( $1^{\circ}$ Ed.).Washington. DC: APA.

Ángel, L. A., Vásquez, R., Martínez, L. M., Chavarro, K., \& García, J. (2000). Comportamiento alimentario: desarrollo, validez y fiabilidad de una encuesta. Revista Colombiana de Psiquiatría, 24, 29-48. Recuperado de http://www.elsevier.es/sites/default/files/elsevier/pdf/27/27v35n02a 13071915pdf001.pdf

Arrivillaga, M., Cortés, C., Goicochea, V., \& Lozano, T. (2003). Caracterización de la depresión en jóvenes universitarios. Universitas Psychologicas, 3 (1) 17-26. Recuperado de http://www.scielo.cl/scieloOrg/php/reflinks.php?refpid=S07184808200900010000100002\&pid=S0718-48082009000100001\&lng=es

Azevedo, M. H., Bento, C., Bos, S., Gomes, A., Macedo, A., Maia, B., Marques, M., Pereira, A. T., Soares, M. J., \& Valente, J. (2010). Perfectionism and eating behavior in portuguese adolescents. European Eating Disorders, 18(4): 328-337. doi: 10.1002/erv.981

Bardone, A. M., \& Fitzsimmonsa, E. E. (2011). Coping and social support as potential moderators of the relation between anxiety and eating disorder symptomatology. Eating Behaviors, 12 (1) 21-28. doi:10.1016/j.eatbeh.2010.09.002

Beato - Fernández, L., \& Rodríguez-Cano, T., (2005). Gender differences regarding psychopathological, family and social characteristics in adolescents with abnormal eating behavior. Eating Behaviors, 6 (4) 337-344. doi: 10.1016/j.eatbeh.2005.02.002 de que éste sea un estudio de corte transversal, esto en términos de las conclusiones referidas a relaciones de causa y efecto limita el alcance de los resultados.

Igualmente, en próximos estudios es conveniente tener en cuenta otros factores como el estrato socioeconómico, el comportamiento alimentario de las personas y de los familiares, el perfeccionismo, los estilos de vida y la participación en actividades deportivas, factores que pueden afectar el riesgo de TCA, tal como lo evidencian algunos estudios preliminares. Adicionalmente, no se puede pasar por alto el hecho de que la medida de TCA (el EAT), si bien identifica comportamientos bulímicos, en sí no está discriminando el riesgo frente a un tipo de trastorno (anorexia o bulimia), por lo que sería importante considerar hacer un modelo predictivo de riesgo para cada Trastorno de la Conducta Alimentaria. En este orden, respecto a la medida de TCA, estudios anteriores realizados en Manizales, Medellín y Cali, muestran un buen comportamiento de la prueba y diferencias significativas entre las regiones, tal como ocurrió con los datos de este estudio entre las ciudades de Ibagué y Bogotá, por lo que valdría la pena pensar en la construcción de baremos para cada región.

Bolaños, Ríos. P., \& Jáuregui. L, I. (2010). Body image and quality of life in a spanish Population. International Journal of General Medicine, 4, 63-72. doi: 10.2147/IJGM.S16201

Buckner, J. D., Schmidt, N. B., Silgado, J., \& Timpano, K. R. (2010). Social anxiety and bulimic behaviors: The moderating role of perfectionism. Cognitive Therapy and Research, 34(5), 487-492. doi: 10.1007/s106080099278-2

Cano, A., Castaño, J., Corredor, D., García, A., González, M., Lloreda, O., Lucero, K., Ortíz, J., Páez, M., Patiño, L., Pérez, L., Villegas, C., \& Zuluaga, A. (2007). Factores de riesgo para trastornos de la alimentación en los alumnos de la Universidad de Manizales. Med UNAB, 3, 187-194. Recuperado

http:/ / revistas.unab.edu.co/index.php?journal $=$ medunab\&page $=$ article \&op= viewArticle\&path $\% 5 \mathrm{~B} \% 5 \mathrm{D}=97$

Carver, C., \& Connor-Smith J. (2010). Personality and Coping. Annual Review of Psychology, 61, 679 - 704 . doi: 10.1146/annurev.psych.093008.100352

Castrillón, D., Luna, L., \& Aguirre-Acevedo, D. (2007). Validación del Abbreviated eating attitudes test (escala abreviada y modificada de las actitudes alimentarias) EAT-26-M para la población colombiana. En: Ferrer, A. y Gómez, Y. (Eds.), Evaluación e intervención en niños y adolescentes: Investigación y conceptualización. (pp. 93-116). Medellín (Colombia): La Carreta Editores.

Castro, M.J. (2002). Anorexia Nerviosa. Guías Clinicas, 2, 1-4. Recuperado de http://www.elsevier.es/sites/default/files/elsevier/pdf/37/37v68n01a 13114466pdf001.pdf

Çatikkas, F. (2011). Physical correlates of college students' body Image satisfaction levels. Social Behavior and Personality, 39(4), 497-502. doi: 10.2224/sbp.2011.39.4.497.

Chico, E. (2002). Optimismo disposicional como predictor de estrategias de afrontamiento. Psicothema, 14, 544-550. Recuperado de http://156.35.33.98/reunido/index.php/PST/article/view/7982/7846

Chinchilla, A. (2005). Trastornos de la conducta alimentaria. Anorexia y bulimia nerviosa, obesidad y atracones. España: Masson.

Conde, T., \& Usereos, E. (1996). Revisión crítica de la adaptación castellana del cuestionario de Beck. Revista Psicología General Aplicada, 31, 469-497. Recuperado 
http:/ /www.scielo.org.co/scielo.php?script $=$ sci_nlinks\&ref $=000080 \& p$ id $=$ S1657-9267200900010000800021\&lng=en

Corstorphine, E., Mountford, V., Tomlinson, S., Waller, G., \& Meyer, C. (2007). Distress tolerance in the eating disorders. Eating Behaviors, 8, 9197. doi: 10.1016/j.eatbeh.2006.02.003.

Crow, S., Eisenberg, M., Story, M., \& Neumark-Sztainer, D. (2006). Psychosocial and behavioral correlates of dieting among overweight and non-over weight adolescents. Journal of Adolescent Health, 38, 569-574. doi: 10.1016/j.jadohealth.2005.05.019,

Dancyger, I., Fornaria, V., Sciontic, L., Sundaya, S., \& Wisotskya, W. (2009). Do daughters with eating disorders agree with their parents' perception of family functioning? Comprehensive Psycbiatry, 10 (1), 22-28. doi: http://dx.doi.org/10.1017/S003329170003751X

De la Fuente, M., García, C., \& Salvador, M. (2009). Creencias y estrategias para el control del peso, satisfacción con la imagen corporal y autoestima. European Journal of Education and Psychology, 3 (2), 257-273. Recuperado

de http://www.formacionasunivep.com/ejep/index.php/journal/article/v iewArticle/ 64

Echeburúa, E., \& Marañón, I. (2001). Comorbilidad de las alteraciones de la conducta alimentaria con los trastornos de la personalidad. Psicología Conductual, 9, 513-525. Recuperado de http://www.companiamedica.com/libros_gratis/salud_mental/trastor nos $\% 20 \mathrm{de} \% 20$ conducta $\% 20$ alimentaria/comorbilidad_alteraciones_co nducta_alimentaria_y_trastornos_.pdf

Edlund, B., Gustafsson, S. A., Kjellin, L., \& Norring, C. (2009). Risk and Protective Factors for Disturbed Eating in Adolescent Girls - Aspects of Perfectionism and Attitudes to Eating and Weight. European Eating Disorders, 17 (5) 380-389. doi: 10.1002/erv.885

Engler, P. A., Crowther, J. H., Dalton, G., \& Sanftner, J. L. (2006). Predicting Eating Disorder Group Membership: An Examination and Extension of the Sociocultural Model. Behavior Therapy, 37, 69-79. doi: 10.1016/j.beth.2005.04.003

Fandiño, A., Giraldo, S., Martínez, C., Aux, C., \& Espinosa, R. (2007). Factores asociados con los trastornos de la conducta alimentaria en estudiantes universitarios en Cali, Colombia. Colombia Medica, 38, 344-351. Recuperado de http://www.scielo.org.co/scielo.php?pid=S165795342007000400002\&script=sci_arttext

Gamboa, E., López, N., \& Quintero, D. (2007). Prevalencia de sobrepeso y obesidad en adolescentes del municipio de Floridablanca, Colombia. Med UNAB, 10, 5-12. Recuperado de http: / $/$ revistas.unab.edu.co/index.php?journal $=$ medunab\&page $=$ article \&op $=$ viewArticle\&path $\% 5 \mathrm{~B} \% 5 \mathrm{D}=120$

Garnefski, N., \& Kraaij, V. (2002). Relationships between cognitive emotion regulation strategies and depressive symptoms: A comparative study of five specific samples. Journal of Adolescent Health, 30, 326-335. doi: 10.1016/j.paid.2005.12.009

Garner, D. M., Olmsted, M. P., Bohr, Y., \& Garfinkel, P. E. (1983). The Eating Attitudes Test: An index of the symptoms of anorexia nervosa. International Journal of Eating Disorders, 2, 15-34. doi: $10.1017 / \mathrm{S} 0033291700049163$

Ghaderi, A. (2003). Structural modeling analysis of prospective risk factors for eating disorder. Eating Behaviors, 3, 387-396. doi: 10.1016/S14710153(02)00089-2

Giovazolias, T., \& Koskina, N. (2010). The Effect of Attachment Insecurity in the Development of Eating Disturbances across Gender: The Role of Body Dissatisfaction. The Journal of Psychology, 144(5), 449-471. doi: 10.1080/00223980.2010.496651

Gómez-Peresmitré, G., \& Acosta, M.V. (2002). Valoración de la delgadez. Un estudio transcultural (México/España). Psicothema, 14, 221-226. http://www.unioviedo.es/reunido/index.php/PST/article/view/8007 $/ 7871$

González, A. N., Oudhof Van. B. H. Rodríguez. A. B., \& Unikel. S. C. (2010). Desarrollo y validación de una escala para medir imagen corporal en mujeres jóvenes. Salud Mental, 33 (4), 325-332. Recuperado de http://www.scielo.org.mx/scielo.php?pid=S018533252010000400004\&script=sci_arttext

Grylli, V., Wagnera, G., Hafferl-Gattermayera, A., Schoberb, E., \& Karwautz, A. (2005). Disturbed eating attitudes, coping styles, and subjec- tive quality of life in adolescents with Type 1 diabetes. Journal of Psychosomatic Research, 59, 65- 72. doi: 10.1016/j.jpsychores.2005.02.010

Hampel, P., \& Petermann, F. (2006). Perceived stress, coping, and adjustment in adolescents. Journal of Adolescent Health, 38, 409-415. doi: 10.1016/j.jadohealth.2005.02.014,

Jaser, S., Champion, J., Reeslund, K., Keller, G., Merchant, M.J., Benson, M., \& Compas, B. (2007). Cross-situational coping with peer and family stressors in adolescent offspring of depressed parents. Journal of Adolescence, 30, 917-932. doi: 10.1016/j.adolescence.2006.11.010

Kluck, A. (2010). Family influence on disordered eating: The role of body image dissatisfaction. Body Image, 7(1), 8-14. doi: 10.1016/j.bodyim.2009.09.009.

Lara, D., \& Akiskal, H. (2006). Toward an integrative model of the spectrum of mood, behavioral and personality disorders based on fear and anger traits: II. Implications for neurobiology, genetics and psychopharmacological treatment. Journal of Affective Disorder, 94 (13), 89-103. doi: 10.1016/j.jad.2006.03.021.

Lazarus, R., \& Folkman, S. (1986). Estrés y Procesos Cognitivos. Madrid, España: Martínez Roca.

Lee, S. K. (2005). Validity of self-reported weight and height: Comparison between immigrant and non-immigrant Mexican Americans in NHANES III. Journal Inmigrant Health, 7(2), 127-31. doi: 10.1007/s10903-005-2646-4

León, M. T., \& Castillo, M. D. (2005). Trastornos de comportamiento alimentario: Anorexia y bulimia nerviosa. Madrid, España: Formación Alcalá.

López, C., Molano, J., \& Piñeros, S., (2010). Factores de riesgo de los trastornos de la conducta alimentaria en jóvenes escolarizados en Cundinamarca (Colombia). Revista Colombiana de Psiquiatría, 39 (2) 313-328. Recuperado

http://www.scielo.org.co/scielo.php?script=sci_arttext\&pid=S003474502010000200007\&lng=en\&nrm=iso >. ISSN 0034-7450

Londoño, C., \& Archila, D. (2004). Consumo de cigarrillo y estilos de afrontamiento. Avances en Psicología. Universidad Santo Tomás, 1, 18-25.

Londoño, C., Hernández, L., Alejo, I., \& Pulido, D. (2013). Diseño y validación del cuestionario de optimismo disposicional para jóvenes. Universitas Psychologica, 12 (1), $01-25$.

Lugli, Z. R., \& Vivas, E. (2006). Trastornos de alimentación, factores socioculturales, creencias de control y dietas. Cuadernos de Medicina Psicosomática y Psiquiatría de Enlace, 79, 23-32. Recuperado de http://www.editorialmedica.info/archivos/cuadernos/Cuad $\% 20 \mathrm{~N} \% \mathrm{C}$ 2\%BA\%2079_80-trab-2.pdf

Maor, N. R., Sayag, S., Dahan, R., \& Hermoni, D. (2006). Eating Attitudes among Adolescents. IMAJ, 8, 627-629. Recuperado de http://www.ima.org.il/imaj/ar06sep-8.pdf

Markey, M.A., \& Vander, J.S. (2007). The role of emotional intelligence and negative affect in bulimic symptomatology. Comprehensive Psychiatry, 48, 458-464. doi: 10.1016/j.comppsych.2007.05.006,

Martínez-González, M. A., \& De Irala, J. (2003). Los trastornos de comportamiento alimentario en España: ¿Estamos preparados para hacerles frente desde la salud pública? Gaceta Sanitaria, 17. Recuperado de http://scielo.isciii.es/scielo.php?pid=S021391112003000500001\&script $=$ sci_arttext\&tlng $=\mathrm{e}$

Mercado, C. (2008). Aplicación del análisis de género a los trastornos de la conducta alimentaria. Revista de Salud, 4, 14. Recuperado de http://www.revistaesalud.com

Ministerio de Protección Social (2003). Un panorama nacional de la salud y enfermedad mental en Colombia: Estudio Nacional de Salud Mental. República de Colombia.

Millar, W. (1991). A trend to a health their life's style. Health Reports, 3, 363 370. PMID:1818734

Moreno, M., \& Ortiz, G. (2009). Trastornos de la Alimentación y su Relación con Imagen Corporal y Autoestima en Adolescentes. Terapia Psicológica, 27 (2), 181-189. doi: 10.4067/S0718-48082009000200004

Moreno, S., Ortega, B., \& Rodríguez, S. (2009). Impulsividad en mujeres con bulimia nerviosa. EduPsykhé, 8 (1), 63-77. Recuperado de http:// + Impulsividad + en + mujeres + con + bulimia + nerviosa. + EduPsykh $\% \mathrm{C} 3$ $\%$ A $9 \% 2 \mathrm{C}++8+\% 281 \% 29+63-77$. $\& b \operatorname{tnG}=\& h l=$ en\&as_sdt $=0 \% 2 \mathrm{C} 5 \&$ as_vis $=1$

Ochoa, I., Espina, A., \& Ortego, M. (2006). Un estudio sobre personalidad, ansiedad y depresión en padres de pacientes con un trastorno alimenta- 
rio. Clínica y Salud, 17, 151-170. Recuperado de http://scielo.isciii.es/scielo.php?pid=S1130-

$52742006000200002 \&$ script $=$ sci_arttext

Organización Mundial de la Salud OPS (2005). Encuesta Nacional de Alimentación y Nutrición, ENAN. 2, 103-106.

Ouwehand, C., De Ridder, D., \& Bensing, J. (2008). Individual differences in the use of proactive coping strategies by middle - age and older adults. Personality and Individual Differences, 45, 28-33. doi: 10.1016/j.paid.2008.02.013.

Palmer, B. (2008). Epidemiology, diagnosing, and assessing eating disorder. Psychiatric, 7 (4) 143-146. doi: 10.1016/j.mppsy.2008.02.005.

Pardo, G., Sandoval, A., \& Umbarila, D. (2004). Adolescencia y depresión. Revista Colombiana de Psicologia, 13, 13-28. Recuperado de http:/ / dialnet.unirioja.es/servlet/articulo?codigo $=3246301$

Peláez, M. A., Labrador, F., \& Raich, R. M. (2005). Prevalencia de los trastornos de la conducta alimentaria: Consideraciones metodológicas. International Journal of Psychology and Psychological Therapy, 5, 135-148. Recuperado

http://www.Prevalencia +de+los + trastornos + de + la + conducta + alimen taria $\% 3 \mathrm{~A}+$ Consideraciones + metodol $\% \mathrm{C} 3 \% \mathrm{~B} 3$ gicas. + International $+\mathrm{Jo}$ ur-

nal + of + Psychology + and + Psychological + Therapy $\% 2 C+\& b \operatorname{tnG}=\& h l=$ en\&as_sdt $=0 \% 2 \mathrm{C} 5$ \&as_vis $=1$

Pérez, S., \& Romero, G. (2008). Imagen corporal en mujeres rurales de la Sierra Juárez y la costa de Oaxaca: una aproximación nutrioantropológica. Instituto Nacional de Ciencias Médicas y Nutrición Salvador Zubirán, 9-32. Recuperado de http://www.scielo.org.mx/scielo.php?script=sci_arttext\&pid=S003636342010000200002

Reba-Harrelson, L., Von Holle, A., Hamer, R., Torgersen, L., ReichbornKjennerud, T., \& Bulik, C. (2010). Patterns of maternal feeding and child eating associated with eating disorders in the Norwegian Mother and Child Cohort Study (MoBa). Eating Behaviors, 11, 54-61. doi: 10.1016/i.eatbeh.2009.09.004.

Renk, K., \& Creasey, G. (2003). The relationship of gender, gender identity, and coping strategies in late adolescents. Journal of Adolescence, 26, 159168. doi: 10.1016/S0140-1971(02)00135-5.

Rivarola, M. F., \& Penna, F. (2006). Los factores socioculturales y su relación con los trastornos alimentarios y su imagen corporal. Revista Intercontinental de Psicología y Educación, 8, 61-72. Recuperado de http://redalyc.uaemex.mx/redalyc/html/802/80280205/80280205.htm 1

Rivarola, M. F. (2003). La imagen corporal en adolescentes mujeres: su valor predictivo en trastornos alimentarios. Fundamentos en Humanidades, 4, 149-161. Recuperado

http://dialnet.unirioja.es/servlet/articulo?codigo $=1273106$

Santacoloma, A., \& Quiroga. B. (2009). Perspectivas de estudio de la conducta alimentaria. Revista Iberoamericana de Psicología: Ciencia y Tecnología, 2 (2) 7-15. Recuperado de https://iberoamericana.edu.co/Publicaciones/RevistaIberoPsicologia/ R03_ARTICULO1_PSIC.pdf
Sepúlveda, A., Botella, J., \& León, J.A. (2001). La alteración de la imagen corporal en los trastornos de la alimentación: Un meta-análisis. Psicothema, 13, 7-16. Recuperado de http://156.35.33.98/reunido/index.php/PST/article/view/7840

Steiger, H. (2007). Eating Disorder Paradigms for the New Millennium: Do "Attachment" and "Culture" Appear on Brain and Genome Scans? Canadian Journal of Psychiatry, 52, 209. Recuperado de http://psycnet.apa.org/psycinfo/2007-10109-001

Solis, C., \& Vidal, A. (2006). Estilos y estrategias de afrontamiento en adolescentes. Revista de Psiquiatría y Salud Mental Hermilio Valdizan, 6, 33-39. Recuperado http:/ / www.scielo.br/scielo.php?script=sci_nlinks\&ref=000144\&pid= S1984-0292201000010000300031\&lng=e

Taylor, S.E., \& Stanton, A.L. (2007). Coping resources, coping processes, and mental health. Annual Review of Clinical Psychology, 3, 377-401. doi: 10.1146/annurev.clinpsy.3.022806.091520

Tusaie, K., \& Patterson, K. (2006). Relationships Among Trait, Situational, \& Comparative Optimism: Clarifying Concepts for a Theoretically Consistent and Evidence-Based Intervention to Maximize Resilience. Arcbives of Psychiatric Nursing, 20, 144-150. doi: 10.1016/j.apnu.2005.10.004

Twohing Unikel, C., \& Gómez-Peresmitré, G. (2004). Validez de constructo de un instrumento para la detección de factores de riesgo en los trastornos de la conducta alimentaria en mujeres Mexicanas. Salud Mental, 27, 38-49. http://www.medigraphic.com/pdfs/salmen/sam2004/sam041e.pdf

Us Department of Health and Human Services \& National Institute of Mental Health (2005). Los hombres y la depresión.

Vázquez, C., \& Sanz, J. (1991). Fiabilidad y validez factorial de la versión española del Inventario de Depresión de Beck. Comunicación presentada en el III Congreso de Evaluación Psicológica, Barcelona, septiembre. Recuperado http://www.unioviedo.es/reunido/index.php/PST/article/view/7467

Velasco, M., Botero, P., \& Londoño, C. (2011). Recursos psicológicos: Afrontamiento, Optimismo y Calidad de Vida. En C. Londoño (Eds.), Avances y perspectivas en psicología de la salud. Colegio Colombiano de Psicólogos Colombia.

Vitaliano, P. P., Russo, J., Carr, J. E., Maiuro, R. D., \& Becker, J. (1985). The Ways of Coping Checklist: Revision and Psychometric Properties. Multivariate Behavioral Research, 20, 3-26. doi: 10.1207/s15327906mbr2001_1

Waller, G., \& Sheffield, A. (2008) Causes of bulimic disorders. Psychiatry, 7, 152-155. doi: 10.1016/j.mppsy.2008.02.002

Watt, M., \& Ricciardelli, L. (2012). A qualitative study of body image and appearance among men of Chinese ancestry in Australia. Body Image, 9, (1) 118-125.doi: 10.1016/j.bodyim.2012.10.002

Welch, E., Miller, J., Ghaderi, A., \& Vaillancourt, T. (2009). Does perfectionism mediate or moderate the relation between body dissatisfaction and disordered eating attitudes and behaviors? Eating Behaviors, 10, 168175. doi: $10.1016 /$ j.eatbeh.2009.05.002

(Articulo recibido: 15-2-2011; revisado: 6-1-2013; aceptado: 13-1-2013) 